\title{
The Intriguing Crystallography of the Simplest Nitriles, Relevant as Putative Cryominerals on Titan, Saturn's Moon \\ T Runcevski ${ }^{1}$ \\ ${ }^{1} S M U$ \\ truncevski@smu.edu
}

Titan, Saturn's icy moon, is an ideal planetary body to study prebiotic chemistry, origins of life, and the potential habitability of an extraterrestrial environment. It features a nitrogen-based atmosphere, complex organic chemistry fueled by radiation from the sun and Saturn's magnetosphere, ethane-based lakes on top of water-ice surface on the poles, organic dunes on the equator, and seasonal evaporation and precipitation of hydrocarbons in a process notably similar to Earth's hydrological cycle. As part of NASA's New Frontiers Mission, a rotorcraft named Dragonfly will be launched in 2027 and is expected to arrive on the surface of Titan in 2034. In light of the imminent Dragonfly mission, revisiting the fundamental crystallography of the smallest organic molecules that make-up the surface of Titan is of utmost importance. Structural research of the smallest organic molecules at cryogenic conditions used to be on the forefront of science following the discovery of X-ray diffraction. Throughout the 20th century, the research field remained active, however, the accent was slowly moved to more complicated molecules and chemistries. Rather surprisingly, there still are important questions on the solid-sate chemistry and crystallography of the smallest molecules that remain unanswered. Here, we present our findings derived from laboratory modeling of the composition and structure of small nitriles as putative Titanean minerals, based on synchrotron X-ray and neutron crystallography. 